Revista de Economia Política, vol. 30, $n^{\circ} 3$ (119), pp. 401-419, julho-setembro/2010

\title{
Fluxo de empregos, fluxo de trabalhadores e fluxo de postos de trabalho no Brasil
}

\author{
EDUARDO PONTUAL RIBEIRO*
}

Job flows, workers flows and employment flows in Brazil. The labor market is in constant flux. When measuring labor market mobility, it is usual to estimate labor market flow statistics, such as worker reallocation and job reallocation. The goal of this paper is to present previously unknown within plant job reallocation statistics for Brazil. The within plant job reallocation is measured as changes in the occupational mix of the plant. The within plant job reallocation allows a decomposition of worker turnover decomposition into matching, technological change and labor demand factors. Using formal labor market data for Brazil, from 1996 to 2001, our results suggest that technological change accounted for a limited share of worker turnover. More than a quarter of worker turnover can be attributed to labor demand shifts and more than $50 \%$ of worker turnover is due to matching.

Keywords: job flows; turnover; technical progress.

JEL Classification: J6.

\section{INTRODUÇÃO}

Recentemente, grande parte da literatura econômica internacional tem focado o estudo da dinâmica dos trabalhadores e de postos de trabalho, pois tal conhecimento é indispensável para a compreensão do mercado de trabalho, com implicações macro e microeconômicas. Um dos interesses em estudar este fluxo de trabalhadores entre estados no mercado de trabalho ou entre empresas é verificar a

\footnotetext{
* Instituto de Economia da Universidade Federal do Rio de Janeiro - IE/UFRJ e Pesquisador do CNPq. E-mail: eribeiro@ie.ufrj.br. Este trabalho faz parte de uma pesquisa sobre realocação no mercado de trabalho, feita em conjunto com técnicos do IPEA e com apoio do CNPq, desde 2003. Agradeço ao parecerista pela melhora significativa do texto, a Carlos Henrique Corseuil pela gratificante troca de ideias, operacionalização das estimativas e explicações quanto aos dados, e sugestões e comentários dos participantes do Encontro da ANPEC 2007 e seminários na UFPR e UFJF. Os erros são de minha inteira responsabilidade. Submetido: Outubro 2008: Aprovado: Março 2009.
} 
capacidade de realocação de recursos de uma economia. Um mercado de trabalho muito flexível pode sugerir maior eficiência alocativa, mas pode gerar grande insegurança para os trabalhadores, pela redução do tempo de permanência em um emprego ou perda de bem-estar por custos de ajustamento e de oportunidade, além de perdas salariais dos trabalhadores na troca do emprego. Outro interesse reside na construção de fatos estilizados para teorias econômicas, com implicações para o papel das recessões e das inovações tecnológicas no ciclo econômico. Um terceiro, mas não menos importante, é a compreensão das diferenças entre taxas de desemprego em diferentes países.

No Brasil, os fluxos de trabalhadores e postos de trabalho são muito altos, como indicam Corseuil e Servo (2006) e Hoek (2002). O mercado de trabalho brasileiro é classificado por alguns estudiosos como "hiperativo" (WorldBank, 2002; Gonzaga, 2003). As taxas de rotatividade e realocação então entre as maiores do mundo, mesmo controlando por outros fatores como composição setorial (indústria, comércio e serviços) e distribuição de tamanhos das empresas.

A literatura iniciada por Davis e Haltiwanger (1992), pelo lado de fluxos de emprego (job flows), e Blanchard e Diamond (1990), pelo lado dos fluxos de trabalhadores (worker flows), tenta entender a dinâmica do mercado de trabalho com interesse último em identificar os componentes do desemprego, seja por choques de demanda (job flows) ou de oferta (worker flows). A coexistência de grandes fluxos de entrada e saída de trabalhadores ao longo de todo o ciclo econômico fomentou a literatura de matching (ou vínculos), como em Mortensen e Pissarides (1999), por exemplo, para entender o desemprego.

De um ponto de vista de uma taxonomia, a rotatividade no mercado de trabalho, ou seja, o volume de admissões e desligamentos na economia, pode ser decomposta em três componentes (Hamermesh et al., 1996). Primeiro: admissões e desligamentos induzidos por ajustes no tamanho da força de trabalho de uma empresa; segundo: a rotatividade induzida por mudanças na estrutura ocupacional da força de trabalho da empresa; e por último, uma parte friccional, respectiva à troca de trabalhadores em postos existentes, em geral por questões de vínculo (match) insatisfatório entre o trabalhador e a empresa.

O primeiro componente destes fluxos de trabalhadores pode ser associado a questões de demanda por emprego e o nível de atividade da economia/empresa. O segundo componente, a questões de mudança tecnológica. E o terceiro, a fatores de oferta de trabalho (quando a dissolução do vínculo entre trabalhador e empresa parte do trabalhador em busca de melhor ocupação) e de match entre as habilidades dos trabalhadores e dos postos de trabalho.

Esta taxonomia explora mensurações com diferentes graus de identificação dos trabalhadores nas bases estatísticas. A partir de dados que identifiquem trabalhadores, características de seus postos de trabalho, e empresas, passa a ser possível estudar esta taxonomia da rotatividade.

Detalhando os conceitos, fluxos de trabalhadores representam as movimentações de pessoas no mercado de trabalho, seja entre empresas, ou entre a atividade, inatividade e desemprego; fluxos de postos de trabalho representam as mudanças 
nas oportunidades de emprego, em que cada emprego está associado a um rol de tarefas e com isto diferenciado através de uma classificação de ocupações; e por fim, os fluxos de emprego medem as mudanças de oportunidades de emprego nas unidades econômicas, em que cada emprego está diferenciado apenas quanto à localização, ou seja, em empresas diferentes. Dito de outra forma, a partir de dados de trabalhadores identificados por ocupações e empresas, os fluxos de trabalhadores medem as variações de estoques de pessoas, em seus postos e empresas; os fluxos de postos de trabalho agrupam as pessoas em ocupações nas empresas, medindo as variações brutas de estoques destas ocupações, em empresas diferentes; e os fluxos de emprego agrupam as ocupações (e pessoas) nas empresas, mensurando as variações brutas de estoques de trabalhadores entre empresas.

Uma compreensão da contribuição de cada parcela para a rotatividade total é indispensável para identificar as fontes da rotatividade e assim desenhar políticas públicas eficientes de redução da rotatividade. Todavia a literatura, particularmente de fluxos de empregos, sintetizada em Davis, Haltiwanger e Schuh (1996), parte de uma definição limitada destes fluxos, em muito restrita pela disponibilidade de dados. Os trabalhos de fluxos de empregos tomam a força de trabalho de uma empresa como homogênea e estudam os fluxos brutos na economia, ou seja, a criação e destruição de emprego.

São muito poucos os trabalhos que conseguem distinguir as mudanças de emprego, postos de trabalho e trabalhadores em uma economia. Inicialmente, apenas Hamermesh et al. (1996) e Anderson e Meyer (1994), explicitam as diferenças entre as três. Outros trabalhos, citados em Abowd, Corbel e Kramarz (1999), relacionam rotatividade com realocação, sem explicitar as mudanças nos postos de trabalho. Para o Brasil, Corseuil e Servo (2006) apresentam uma visão exaustiva da realocação de emprego, sem tratar de rotatividade ou realocação de postos de trabalho. Do ponto de vista teórico, um trabalho na linha de pesquisa deste artigo é Corseuil (2008), em que a realocação de postos de trabalho é induzida por investimentos na firma. Corseuil (2007) e Corseuil e Hayashi (2006) também estudam a realocação de postos de trabalho e de emprego para o Brasil, mas empregando outra definição de realocação de postos de trabalho, condicional à rotatividade e uma decomposição de rotatividade diferente da apresentada aqui. Outro trabalho próximo ao nosso é Orellano e Pazello (2006). As autoras estudam a relação entre rotatividade e realocação de emprego para a Região Metropolitana de São Paulo e definiram uma medida chamada churning líquido, usando três categorias educacionais.

Nosso trabalho diferencia-se por várias razões: primeiro, porque consideramos explicitamente os postos de trabalho classificados por ocupações — categorização mais detalhada que escolaridade —; segundo, porque apresentamos resultados para o Brasil como um todo; e terceiro, pois explicitamos a derivação da decomposição da rotatividade e sinalizamos inter-relações entre estatísticas. Por fim, sob hipóteses, atribuímos uma interpretação funcional à decomposição da rotatividade proposta.

Políticas públicas eficientes que visem tratar a rotatividade, o tempo de emprego e o desemprego, tanto do ponto de vista microeconômico, como macroeconômicas beneficiam-se dos resultados. Por exemplo, se o problema do desemprego 
for de grande destruição de postos de trabalho, políticas de geração de emprego (pela criação de empresas) serão menos eficientes que políticas de sustentação de postos de trabalho existentes, focando as empresas que já existem. Se grande parte da rotatividade for devida a mudanças na estrutura ocupacional, políticas de inovação e desenvolvimento industrial — como a atual Política de Desenvolvimento Produtivo (PDP) do Governo Federal — devem ser acompanhadas por políticas não só de treinamento, mas também de recolocação e reciclagem de trabalhadores, que serão desligados de seus postos pela inovação tecnológica.

Este artigo se divide em seis partes, inclusive esta introdução. Na próxima seção definem-se os conceitos de fluxos de trabalhadores, postos de trabalho e emprego. A seção seguinte apresenta suas inter-relações e a decomposição básica para identificar as fontes da rotatividade. A penúltima seção apresenta as estimativas e a última traça alguns comentários finais.

\section{FLUXOS DE TRABALHADORES, POSTOS DE TRABALHO E EMPREGOS: ASPECTOS CONCEITUAIS ${ }^{1}$}

Como dito na introdução, para discutir os fluxos do mercado de trabalho faz-se necessário determinar os conceitos empregados e suas inter-relações. Muitos trabalhos, por limitações dos dados, usam de modo equivalente "posto de trabalho" e "emprego", quando na verdade estes conceitos são diferentes. Antes, deve-se deixar claro que o uso corrente dos conceitos é confuso na literatura nacional e internacional. Por exemplo, quando um relatório de análise do mercado de trabalho como o do IPEA ou MTE afirma, baseado em pesquisas domiciliares (PME) ou dados administrativos (como o CAGED/RAIS), que tantos postos de trabalho foram criados ou destruídos, de modo líquido, na verdade tal aumento deve-se a mudanças líquidas no número de ocupados. Implicitamente, supõe-se que não há mudanças na estrutura ocupacional nas empresas, excedentes a mudanças líquidas do emprego, não existem vagas desocupadas em nenhum momento e que empregadores e "conta próprias" são contabilizados como ocupantes de postos de trabalho. Estas hipóteses são claramente irrealistas.

\section{Fluxos de trabalhadores medidos pelo lado da empresa ou estabelecimento}

Um número considerável de estudos, anterior à literatura de criação e destruição de emprego, preocupa-se com fluxos de trabalhadores (transições) entre estados no mercado de trabalho. ${ }^{2}$ Como dito acima, pode-se identificar três situações no mercado de trabalho: pessoa ocupada ou empregada, $E$; pessoa desempregada (sem ocupação, mas procurando emprego), $U$; e pessoa fora do mercado de trabalho, ou

\footnotetext{
${ }^{1}$ Esta seção baseia-se em parte em Corseuil e Servo (2006).

${ }^{2}$ Vide Ehrenberg e Smith (2000), entre outros, e no Brasil, Neri et al.(1996).
} 
seja, nem empregada nem procurando emprego, O. A análise destes fluxos de trabalhadores refere-se à identificação dos estoques nas três situações no mercado de trabalho e suas inter-relações. Este tipo de análise pressupõe o uso de pesquisas domiciliares, que não é o caso aqui. Uma pesquisa poderia gerar o seguinte caso hipotético:

Tabela 1: Casos hipotéticos de movimentação de trabalhadores - medição direta.

\begin{tabular}{|c|c|c|c|}
\hline Período & $t-1$ & $T$ & $t+1$ \\
\hline Trabalhador 1 & Empresa 1 & Empresa 2 & Empresa 2 \\
\hline Trabalhador 2 & Empresa 1 & Empresa 1 & Fora da Força de Trab. \\
\hline Trabalhador 3 & Empresa 2 & Desempregado & Desempregado \\
\hline Trabalhador 4 & Desempregado & Empresa 1 & . \\
\hline
\end{tabular}

Nota: medidas feitas em uma data fixa no intervalo (por exemplo, no último dia do mês).

Uma outra visão do cálculo do fluxo dos trabalhadores e movimentação no mercado de trabalho baseia-se em dados das empresas. Por um lado, este tipo de análise é mais limitada que a anterior, já que o destino ou origem dos trabalhadores que ocupam as posições na empresa são desconhecidos ou desconsiderados. Por outro lado, pode-se identificar se o desligamento deveu-se a fatores de oferta ou demanda de trabalho. $\mathrm{Na}$ apresentação, seguiremos $\mathrm{Ha}-$ mermesh et al. (1996).

Os fluxos de trabalhadores pelo lado de uma empresa podem ser divididos em duas partes, admissões e desligamentos, a saber:

$$
\text { Admissões }_{\mathrm{it}}=\mathrm{H}_{\mathrm{it}}+\mathrm{RH}_{\mathrm{it}}+\mathrm{TI}_{\mathrm{it}}
$$

onde H representa contratações (hires), RH recontratações (rehires) e TI transferências de outros estabelecimentos da empresa, e

$$
\text { Desligamentos }_{\mathrm{it}}=\mathrm{Q}_{\mathrm{it}}+\mathrm{F}_{\mathrm{it}}+\mathrm{D}_{\mathrm{it}}+\mathrm{TO}_{\mathrm{it}} \text {, }
$$

onde Q são desligamentos voluntários (quits), F demissões (fires/layoffs), D “discharges for cause", ou seja, dispensas por outros motivos, como aposentadorias, e TO, transferências para outros estabelecimentos da empresa. Desligamentos são denominados também separations (S) na literatura internacional. No caso de dados de uma empresa que tenha um estabelecimento apenas, $\mathrm{TO}_{\mathrm{it}}$ e $\mathrm{TI}_{\mathrm{it}}$ são zero.

Todas estas variáveis, em geral, são medidas como fluxos entre dois períodos, ou seja, total de ocorrências em um intervalo de tempo. Esta visão difere daquela da análise dos trabalhadores, na qual, em geral, os dados são apenas de final de período.

Prosseguindo com nosso exemplo para ilustrar os comentários acima, construímos a Tabela 2 supondo dados de fluxos detalhados dos trabalhadores nas empresas e, no caso específico do exemplo, abstraindo as mudanças intraperíodo destes. 
Tabela 2: Casos hipotéticos de movimentação

de trabalhadores - medição via fluxos de funcionários.

\begin{tabular}{|l|c|c|}
\hline \multicolumn{1}{|c|}{ Período } & $t$ & $t+1$ \\
\hline Empresa $1 \mathrm{H}$ & 1 & 0 \\
\hline $\mathrm{S}$ & 1 & 1 \\
\hline Empresa $2 \mathrm{H}$ & 1 & 0 \\
\hline $\mathrm{S}$ & 1 & 0 \\
\hline
\end{tabular}

Nota: baseado na Tabela 1. $\boldsymbol{H}$-admissões; $\boldsymbol{S}$-desligamentos.

O estoque de empregados em um período $n_{i t}$ pode ser calculado diretamente ou através de

$n_{i t}=n_{i t-1}+$ Admissões $_{i t}-$ Desligamentos $_{i t}=H_{i t}-S_{i t}$.

Baseado na Tabela 1, pode-se construir a Tabela 3, que representa o caso de se ter apenas dados do estoque de funcionários:

Tabela 3: Casos hipotéticos de movimentação

de trabalhadores - medição via estoques de funcionários $\left(n_{i t}\right)$.

\begin{tabular}{|l|c|c|c|}
\hline \multicolumn{1}{|c|}{ Período } & $t-1$ & $t$ & $t+1$ \\
\hline Empresa 1 & 2 & 2 & 1 \\
\hline Empresa 2 & 1 & 1 & 1 \\
\hline
\end{tabular}

Nota: baseado na Tabela 1

Mais uma vez, note que se temos acesso apenas a dados do estoque de empregados $n_{t}$ em dois períodos no tempo, a informação de fluxos de trabalhadores seria impossível de ser inferida. Davis e Haltiwanger (1992) sugerem uma medida alternativa à diferenciação entre admissões e desligamentos, para medir fluxos de empregos, baseada apenas na variação do emprego total da empresa, ou seja, apenas no efeito líquido da mudança no emprego em cada empresa, $\Delta n_{i t}=n_{i t}-n_{i t-1}$. Apesar de subestimar os fluxos de trabalhadores, estas medidas são justificadas por destacar motivações de demanda por emprego para os fluxos de trabalhadores. A (taxa de) criação de emprego (Job Creation, JC) na economia é definida como a soma das variações do emprego daquelas firmas que tiveram crescimento do emprego:

$$
J C_{t}=\sum_{i=1}^{N}\left(\Delta n_{i t} / X_{t}\right) I\left(\Delta n_{i t} \geq 0\right)
$$

onde $I$ ( ) é a função indicador, que toma valor 1 se o critério é verdadeiro e 0 se falso, e $X_{t}$ o estoque médio de pessoas empregadas $X_{t}=\sum_{i=1}^{N}\left(n_{i t}+n_{i-1}\right) / 2$, para as $\mathrm{i}=1, \ldots \mathrm{M}$ empresas da economia.

De modo simétrico, a (taxa de) destruição de emprego (Job Destruction JD) pode ser definida como soma das taxas de crescimento negativas das firmas da amostra: 


$$
J D_{t}=\sum_{i=1}^{N}\left(\left|\Delta n_{i t}\right| / X_{t}\right) I\left(\Delta n_{i t}<0\right)
$$

onde $I\left(\right.$ ) é definido como acima. Note que $J D_{t}$ será positivo, apesar de ser uma soma de valores negativos. A criação (destruição, respectivamente) de empregos, JC $(J D)$ pode ser dividida em duas partes, separando aquela parte relacionada a firmas que começam a operar (que fecham ou saem da amostra), isto é, a entrada (saída) de firmas, e a parte devido a empresas que continuam na amostra, também ditas sobreviventes, isto é, aquelas que possuem emprego positivo nos anos $t$ e $t-1$.

Além dos fluxos de empregos e trabalhadores, com uma base de dados que identifique o tipo de ocupação (atividade) de cada trabalhador é possível medir as variações de postos de trabalho na economia. Por exemplo, no tempo $t$, a empresa 1 ao trocar o trabalhador 1 pelo trabalhador 4 na Tabela 1 pode ter colocado o trabalhador 4 no mesmo posto de trabalho (ocupação), ou em outro posto. Suponha que o posto ocupado pelo trabalhador 1 seja de contador e o posto do trabalhador 4, na mesma empresa, de economista. Neste caso temos a destruição e criação de um posto de trabalho entre os períodos $t-1$ e $t$. Por outro lado, a empresa 2 , em $t$, ao demitir o trabalhador 3 e admitir o trabalhador 1 , pode tê-lo feito para o mesmo posto de trabalho.

Denominando PC (PD, respectivamente), a criação (destruição) de postos de trabalho, temos

$$
\begin{aligned}
& P C_{t}=\sum_{i=1}^{N} \sum_{J=1}^{J}\left(\Delta p_{i t}^{j} / X_{t}\right) I\left(\Delta p_{i t}^{j}<0\right) \\
& P D_{t}=\sum_{i=1}^{N} \sum_{J=1}^{J}\left(\left|\Delta p_{i t}^{j}\right| / X_{t}\right) I\left(\Delta p_{i t}^{j}<0\right)
\end{aligned}
$$

onde $\Delta p^{i}{ }_{i t}=p_{i t}^{j}-p_{i t-1}^{j}$ indica as mudanças nos estoques de postos $j$ na empresa $i$ entre $t-1$ e $t$. Os postos podem ser identificados através de classificações de ocupações como a CBO para o Brasil. Para o exemplo em questão, temos $P C_{t}=1 / 3$ e $P D_{t}=1 / 3$. Já em $t+1$, a empresa 1 pode ter eliminado o posto ocupado pelo trabalhador 4. Assim, $P C_{t+1}=0$ e $P D_{t+1}=1 / 2,5 .^{3}$

Em suma, para o exemplo apresentado, medindo os índices em taxas em função do estoque de emprego médio entre períodos, temos os resultados da tabela 1 para os fluxos de trabalhadores $(h, s)$, emprego $(J C, J D)$ e postos de trabalho $(P C, P D)$.

Tabela 4: Casos hipotéticos de movimentação de trabalhadores, empregos e postos de trabalho - medição via dados de empresas e empregados.

\begin{tabular}{|c|c|c|}
\hline Período & $T$ & $t+1$ \\
\hline$h$ & $2 / 3$ & $0 / 2.5$ \\
\hline$s$ & $2 / 3$ & $1 / 2.5$ \\
\hline$J C$ & $0 / 3$ & $0 / 2.5$ \\
\hline
\end{tabular}

continua na página seguinte

\footnotetext{
${ }^{3}$ Hamermesh et al. (1996) e Dunne et al. (1997) usam outra notação para estas medidas.
} 


\begin{tabular}{|c|c|c|}
\hline$J D$ & $0 / 3$ & $1 / 2.5$ \\
\hline$P C$ & $1 / 3$ & $0 / 2.5$ \\
\hline$P D$ & $1 / 3$ & $1 / 2.5$ \\
\hline$\Delta n$ & 0 & -1 \\
\hline
\end{tabular}

Nota: baseado na Tabela 1 e hipóteses complementares do texto. $h$ - taxa de admissões, $s$ - taxa de desligamentos, $J C$ - taxa de criação de emprego, $J C$ - taxa de destruição de emprego, $P C$ - taxa de criação de postos de trabalho, $P D$ - taxa de destruição de postos de trabalho, $\Delta n$-variação líquida do emprego agregado. $X_{t}=3$ e $X_{t+1}=2.5$.

A variação líquida no estoque de pessoas empregadas na economia pode ser medida de diferentes formas, gerando o mesmo resultado, isto é,

$\Delta \mathrm{n}_{\mathrm{t}}=\mathrm{h}_{\mathrm{t}}-\mathrm{s}_{\mathrm{t}}=\mathrm{JC}_{\mathrm{t}}-\mathrm{JD}_{\mathrm{t}}=\mathrm{PC}_{\mathrm{t}}-\mathrm{PD}_{\mathrm{t}}$

Por outro lado,

$$
h_{t}+s_{t} \geq P C_{t}+P D D_{t} \geq J C_{t}+J D_{t} \geq\left|\Delta n_{t}\right| .
$$

Esta desigualdade básica será explorada para gerar um método de identificação das fontes da rotatividade, como mencionado na introdução e objetivos. Mas, antes de passar para a decomposição, vamos apresentar definições de medidas de "turbulência" ou movimentação no mercado de trabalho.

\section{Medidas de mobilidade e turbulência no mercado de trabalho}

Várias estatísticas de movimentação no mercado de trabalho podem ser construídas a partir das variáveis acima. Nas pesquisas sobre os fluxos no mercado de trabalho, o estudo das variações líquidas muitas vezes não é o mais importante, se existem custos de ajustamento para as transições. Em paralelo ao exemplo acima de $\Delta n_{t}=0$, um aumento líquido no emprego agregado em 5 empregos $\left(\Delta n_{t}=5\right)$ pode ser devido à expansão em cinco vagas em empresas apenas, ou na verdade devido ao desligamento de 100 trabalhadores e 105 admissões. Dados os custos não nulos de ajustamentos, a perda de bem-estar associada à segunda situação pode ser significativa. Para isso é importante estudar o volume bruto de transições no mercado de trabalho e não apenas seu resultado líquido.

Diferentes formas de mensuração do mercado de trabalho, pelo lado da empresa, geram diferentes medidas do volume de transições ou mobilidade no mercado de trabalho, quer se considerem trabalhadores, postos de trabalho ou estoque de emprego.

Talvez a mais comum seja aquela medida pelo lado dos trabalhadores e denominada a rotatividade de trabalhadores (total turnover ou worker turnover), que Davis e Haltiwanger (1995) definem como "o número de admissões e desligamentos que ocorrem no intervalo de t a t-1" (p. 5, tradução nossa). Usando as defini- 
ções da segunda seção e baseado em dados de registros das empresas, podemos medir a rotatividade de trabalhadores como

$$
T_{t}=h_{t}+s_{t}=\sum_{i=1}^{N}\left(h_{i t}+s_{i t}\right)
$$

Davis e Haltiwanger (1995) alegam que a medida (6) indica o número de transições no mercado de trabalho. Note que isto é apenas parcialmente correto, pois as transições entre desemprego $(\mathrm{U})$ e fora da força de trabalho $(\mathrm{O})$ não são consideradas. Ou seja, T mede apenas a movimentação entre ocupação (E) e desocupação (U e O).

Baseado na Tabela 4, temos $\mathrm{T}_{\mathrm{t}}=(2 / 3)+(2 / 3)=4 / 3$ e $\mathrm{T}_{\mathrm{t}+1}=(0 / 2,5)+(1 / 2,5)=1 / 2,5$. Há dupla contagem na medida de rotatividade. No período $t$, três trabalhadores tiveram transições saindo da e obtendo ocupações. Mas a dupla contagem não significa que devemos dividir o valor por dois, pois a mesma é devido a dupla contagem das transições de emprego para emprego apenas.

No Brasil, outra medida de rotatividade popular (usada por Camargo, 1996, Orellano e Pazzello, 2006, e outros) pode ser expressa na equação abaixo:

$\mathrm{T}_{\mathrm{t}}^{\mathrm{BR}}=\operatorname{Min}\left\{\mathrm{h}_{\mathrm{t}}, \mathrm{s}_{\mathrm{t}}\right\} / \mathrm{X}_{\mathrm{t}}$.

Esta medida parte do princípio de substituição, em que a "rotatividade" seria a parcela de admissões e desligamentos que não é necessária para acomodar variações líquidas no emprego. Isto é, $T_{t}^{\mathrm{BR}}=\left(\mathrm{T}_{\mathrm{t}}-\left|\Delta \mathrm{n}_{\mathrm{t}} / \mathrm{X}_{\mathrm{t}}\right|\right) / 2$. Nossa abordagem seguirá, no entanto, a literatura internacional, associando rotatividade com transições no mercado de trabalho. ${ }^{4} \mathrm{O}$ ponto de vista é que qualquer transição pode gerar custos de ajustamento e perdas de bem-estar individuais, e não apenas aquelas que se sobrepõem a variações líquidas do emprego. Outra razão de não usarmos $\mathrm{T}^{\mathrm{BR}}$ é de que $\mathrm{T}^{\mathrm{BR}}{ }_{\mathrm{t}} \neq \sum_{i=1}^{N} w_{i t} \mathrm{~T}^{\mathrm{BR}}{ }_{\mathrm{it}}$, enquanto a agregação é exata para $\mathrm{T} .{ }^{5}$

Já no caso de bases de dados de empresas que apenas apresentam o estoque de empregados em um dado ponto no tempo (Tabela 3), fica impossível medir a rotatividade $T$ ou $T^{\mathrm{BR}}$. Neste caso, Davis e Haltiwanger (1992) apresentam a existência de um limite inferior para tal rotatividade, a taxa de Realocação Bruta de Emprego (Gross Job Reallocation, GJR). Esta taxa é medida como a soma dos dois componentes da mudança de nível de emprego, especificados em (2a) e (2b):

$$
\mathrm{GJR}_{\mathrm{t}}=\sum_{i=1}^{N}\left|\Delta \mathrm{n}_{\mathrm{it}}\right| / \mathrm{X}_{\mathrm{it}}=\mathrm{JC}_{\mathrm{t}}+\mathrm{JD}_{\mathrm{t}}
$$

Esta taxa de realocação é um agregado das mudanças líquidas dos estoques de empregados em cada empresa. Todas as admissões e desligamentos que não alteram o estoque de empregados no período de mensuração não são consideradas, gerando assim um limite inferior para a rotatividade de trabalhadores.

Uma medida da intensidade de rotatividade de emprego pode ser vista definindo a realocação em excesso (Excess Job Reallocation, EJR):

\footnotetext{
${ }^{4}$ Abaixo definiremos uma medida semelhante à $T^{B R}$.

${ }^{5}$ Onde $w_{i t}=x_{i t} / X_{t}$ e $x_{i t}=\left(n_{i t}+n_{i t-1}\right) / 2$.
} 


$$
E J R_{t}=G J R_{t}-\left|\Delta n_{t}\right|=\sum_{i=1}^{N}\left|\Delta n_{t}\right| / X_{i t}-\left|\sum_{i=1}^{N} \Delta n_{t} / X_{i t}\right|
$$

EJR mede quanto da realocação do emprego não pode ser explicada pela necessidade de crescimento (ou redução) líquida do emprego. Se todas as empresas andassem na mesma direção, ou seja, todas reduzissem ou aumentassem o emprego em um dado $t, E J R_{t}$ seria zero. Esta medida é análoga à $T^{B R}$, para o caso da rotatividade de trabalhadores, substituindo GRJ por $T$.

Fica claro que a realocação de empregos é um conceito diferente da rotatividade de trabalhadores, entendido como mudanças de pessoas entre postos de trabalhos. Se o número de admissões e de desligamentos for igual, a mudança no número de postos de trabalho medido através de $G J R_{t}$ é zero, mas a rotatividade de trabalhadores será positiva. Mais ainda, por exemplo, se uma empresa tem uma posição para engenheiro e esta pessoa passa a ser diretor devido à demissão do ocupante anterior da vaga, mas a posição anterior não é reocupada e sim extinta, note que há a destruição de um posto de trabalho, uma promoção e uma demissão. O emprego líquido na empresa cai em uma posição e em um trabalhador, devido à destruição de um posto de trabalho. Mas o oposto nem sempre é verdade. Movimentos na força de trabalho não são motivados apenas por destruição de postos de trabalho, mas também por problemas de qualidade do vínculo (matching) e interesses das empresas.

Em relação à movimentação de postos de trabalho, podemos definir, de modo análogo a $T$ e GJR, uma medida de realocação de postos de trabalho, baseado em medidas de variações de postos de trabalho nas empresas (Dunne et al., 1997). A medida de realocação de postos de trabalho $\left(P R_{t}\right)$ pode ser calculada como

$$
P R_{t}=\sum_{i=1}^{N} \quad \sum_{j=1}^{J}\left|\Delta_{p i t}^{j}\right| / X_{t}=P C_{t}+P D_{t-}
$$

Replicando a equação (5), com a nova notação, temos

$$
\mathrm{T}_{\mathrm{t}} \geq \mathrm{PR}_{\mathrm{t}} \geq \mathrm{GJR}_{\mathrm{t}} \geq\left|\Delta \mathrm{n}_{\mathrm{t}}\right|,
$$

As três medidas serão iguais se a mudança líquida no emprego na economia vier de empresas ajustando o emprego na mesma direção, e sem mudanças nos postos de trabalho (além dos que forem criados ou destruídos, junto com os empregos) e sem substituição de trabalhadores. Se a equação for calculada para uma empresa i específica, note que:

$$
\mathrm{T}_{\mathrm{it}} \geq \mathrm{PR}_{\mathrm{it}} \geq \mathrm{GJR}_{\mathrm{it}}=\left|\Delta \mathrm{n}_{\mathrm{it}} / \mathrm{X}_{\mathrm{it}}\right| .
$$

Como dito na introdução, esta desigualdade (5) será utilizada para identificar as fontes da rotatividade na economia. 


\section{FONTES DA ROTATIVIDADE}

$\mathrm{Na}$ desigualdade descrita acima (eq. 5'), a rotatividade de trabalhadores será sempre maior que a realocação de postos de trabalho (na economia e/ou uma empresa), a realocação de emprego e as variações líquidas de emprego. Primeiro, porque a rotatividade de trabalhadores inclui movimentos de substituição de trabalhadores em postos de trabalho já existentes. Na literatura, a razão principal para esta substituição seria o desejo de desfazer um vínculo empregatício (match), devido à resultados abaixo do esperado (em termos de lucratividade e salários para a empresa e trabalhador, respectivamente) do vínculo (Mortensen e Pissarides, 1999). O trabalhador pode concluir que existe um empregador melhor na economia e/ou o empregador pode pensar que existe um trabalhador melhor para o posto.

Segundo, porque a rotatividade de trabalhadores pode ser induzida por rearranjos produtivos que não necessariamente levem a mudanças similares no estoque de postos de trabalho. O caso dos bancos é típico, em que os "escriturários" que trabalhavam em caixas, ou apoiando "caixas", foram substituídos por terminais de atendimento eletrônico e pessoal de apoio a estas máquinas. Em geral, pode-se atribuir mudanças na estrutura ocupacional a mudanças nos processos produtivos, isto é, progresso técnico.

Terceiro, porque mudanças na demanda dos bens produzidos na empresa alteram apenas o estoque de empregados em uma empresa, ${ }^{6}$ ao invés de gerar uma substituição de trabalhadores e/ou troca de postos de trabalho. A variação no estoque de empregados induz a uma mudança no estoque de ocupações pela extinção ou criação de postos, mas não na sua distribuição relativa.

Assim, podemos propor a seguinte decomposição da rotatividade de trabalhadores:

$$
\begin{aligned}
& T_{t}=\left|\Delta n_{t}\right|+\left(G J R_{t}-\left|\Delta n_{t}\right|\right)+\left(P R_{t}-G J R_{t}\right)+\left(T_{t}-P R_{t}\right) \\
& \text { I II III IV }
\end{aligned}
$$

O primeiro termo do lado direito identifica a parcela da rotatividade que depende de variações líquidas do emprego na economia. Esta parte pode ser associada a variações da rotatividade advindas de choques agregados. O segundo termo identifica a parcela da rotatividade que depende de choques realocativos entre os setores da economia e/ou choques idiossincráticos nas empresas e já foi definido como EJR na seção anterior.

O terceiro termo identifica a parcela da rotatividade que depende de rearranjos produtivos, isto é, mudanças na estrutura ocupacional além das que seriam necessárias para acomodar variações no estoque de empregados das empresas. Este terceiro termo pode ser induzido por outros fatores que não apenas mudança tecnológica. Em particular excluímos mudanças de salários relativos entre catego-

\footnotetext{
${ }^{6}$ Abstraímos aqui mudanças nas horas trabalhadas e/ou na intensidade do trabalho. Isto é padrão na literatura. Veja, por exemplo, Hamermesh et al. (1996) e Davis, Haltiwanger e Schuh (1996).
} 
rias ocupacionais e tecnologia heterotética de produção. No mínimo, esta parcela determina um limite superior para a parcela da rotatividade devido à inovação (entendida como mudança na estrutura ocupacional).

Por último, o quarto termo identifica a parcela de rotatividade que reflete uma pura substituição de trabalhadores entre os postos de trabalho existentes (já desconsideradas as mudanças nos postos induzidos por ajustes no estoque de trabalhadores) e choques agregados. Esta parcela seria devido a questões de vínculo entre empregadores e empregados.

Esta decomposição, contribuição original desta pesquisa, supera de diferentes formas as várias medidas existentes na literatura para compreender a rotatividade de trabalhadores. Burgess et al. (2001) definem como churning $(\mathrm{CH})$ a medida empregada no Brasil como rotatividade $\left(T^{B R}\right)$. Uma medida similar é dada em Albaek e Sorensen (1998) e denominada replacements. Isto é

$$
\mathrm{CH}_{\mathrm{t}}=\mathrm{T}_{\mathrm{t}}-\left|\Delta \mathrm{n}_{\mathrm{it}}\right|_{\mathrm{t}}=\left(\mathrm{h}_{\mathrm{t}}+\mathrm{s}_{\mathrm{t}}\right)-\left|\mathrm{h}_{\mathrm{t}}-\mathrm{s}_{\mathrm{t}}\right|=2 \operatorname{Min}\left\{\mathrm{h}_{\mathrm{t}}, \mathrm{s}_{\mathrm{t}}\right\}
$$

O problema com esta medida é que, como comentam os autores, a medida reflete " $[\ldots]$ trabalhadores partindo para outras oportunidades e sendo substituídos pelos empregadores [...] ou empresas tentando mudar a composição de habilidades da sua força de trabalho"(Burguess et al., 2001, p. 3). ${ }^{7}$ Desta forma, a medida confunde as duas formas de rotatividade, aquela devida à mudança tecnológica $\mathrm{e}$ aquela devida ao matching apenas. Neste trabalho pretendemos contribuir para a literatura separando os efeitos de mudança tecnológica e de matching da rotatividade de trabalhadores. Orellano e Pazzelo (2006) também calculam o churning, mas ele é definido como $T-G J R$. As autoras também estimam $P R-\left|\Delta n_{t}\right|$, sob nome de ERFQ (excesso de realocação entre faixas de qualificação). A definição usada naquele artigo para RL (rotatividade líquida) equivale, em nosso caso, a $T$ $-P R$, ou seja, o termo IV da decomposição acima. Corseuil (2007) também estima medidas similares, mas sem explicitar a decomposição acima.

Uma questão que pode ser levantada é que enquanto as parcelas I, II e III da decomposição (10) dizem respeito a estoques de vínculos, a parcela IV compara o número de trabalhadores com o número de vínculos. Com isto, e seguindo a sugestão de Dunne et al. (1997), pode ser interessante entender a realocação de postos de trabalho apenas, decompondo a realocação de postos de trabalho $(P R)$ em três parcelas. Uma, devido a choques agregados (variação líquida na economia), a segunda devido à realocação entre estabelecimentos (entre unidades) e uma devido à realocação dentro do estabelecimento (intraunidade), ou seja, mudanças na estrutura ocupacional que não implicam mudanças no tamanho da unidade econômica.

$$
\begin{array}{ccc}
P R_{t}=\left|\Delta n_{t}\right|+ & \left(G J R_{t}-\left|\Delta n_{t}\right|\right)+\left(P R_{t}-G J R_{t}\right) \\
\text { li }) & \text { (II) } & \text { (III) } \\
\text { líquido } & \text { entre unidades } & \text { intraunidade }
\end{array}
$$

\footnotetext{
${ }^{7}$ Tradução do autor e ênfase adicionada.
} 
Dois trabalhos na literatura que permitem a decomposição acima (embora não tenham sido feitas explicitamente) são Hamermesh et al.(1996) e Salvanes e Forre (2003). ${ }^{8}$ Como pode ser visto na Tabela 5 abaixo, a maior parte da rotatividade vem de problemas de vínculo entre empresas e empregados, seguido por choques realocativos/idiossincráticos para a Holanda em 1990. A mudança tecnológica apresenta a menor contribuição, sendo superada por choques de demanda (variação líquida do emprego). Em Salvanes e Forre (2003) a mudança tecnológica e choques de demanda também apresentam as menores contribuições, com maior importância para choques realocativos e matching. Os dados deste último trabalho são para a média de 1987 a 1994 para a Indústria de Transformação na Noruega.

Tabela 5: Decomposição da rotatividade — resultados da literatura internacional

\begin{tabular}{|c|c|c|c|c|}
\hline Artigo & $\begin{array}{c}\text { Choques de } \\
\text { demanda (I) }\end{array}$ & $\begin{array}{c}\text { Choques } \\
\text { realocativos (II) }\end{array}$ & $\begin{array}{c}\text { Mudança } \\
\text { tecnológica (III) }\end{array}$ & $\begin{array}{c}\text { Matching } \\
\text { (IV) }\end{array}$ \\
\hline Hamermesh et al. (1996) & 8.18 & 20.00 & 3.64 & 68.18 \\
\hline Salvanes e Forre (2003) & 6.52 & 47.46 & 6.75 & 39.27 \\
\hline
\end{tabular}

Nota: como \% da rotatividade.

Este resultado não deve ser visto como indicador de que o impacto da mudança tecnológica na rotatividade é desprezível em todas as economias. O caso apresentado pode ser excepcional, pois ou cobre apenas um ano específico (no caso da Holanda), em que mudanças tecnológicas podem ter pouco efeito, ou traz resultados para países pequenos e desenvolvidos (Holanda e Noruega), enquanto para o Brasil, como em outros países em desenvolvimento, a rotatividade e realocação de emprego é três vezes maior.

Por outro lado, além dos autores anteriores, Dunne et al. (1997) pode ser usado para a decomposição (12). ${ }^{9}$

Tabela 6: Decomposição da realocação de postos de trabalho na literatura

\begin{tabular}{|l|c|c|c|}
\hline \multicolumn{1}{|c|}{ Artigo } & $\begin{array}{c}\text { Choques de } \\
\text { demanda (I) }\end{array}$ & $\begin{array}{c}\text { Choques } \\
\text { realocativos (II) }\end{array}$ & $\begin{array}{c}\text { Mudança } \\
\text { tecnológica (III) }\end{array}$ \\
\hline Hamermesh et al. (1996) & 25,7 & 62,9 & 11,4 \\
\hline Dunne et al. (1997) & 4,6 & 83,1 & 12,3 \\
\hline Salvanes e Forre (2003) & 10,7 & 78,2 & 11,1 \\
\hline
\end{tabular}

Nota: como \% da realocação de postos de trabalho.

\footnotetext{
${ }^{8}$ Ao trabalhar com realocação em excesso do emprego, os resultados de Orellano e Pazello (2007) não permitem comparação direta com a decomposição 17.

${ }^{9}$ Bauer e Bender (2004) não pode ser usado, pois não apresenta estimativas de GJR, apenas de PR. Corseuil e Hayashi (2006) usam outra definição de PD. Orellano e Pazzelo (2006) não trazem uma tabela de números, apenas gráficos.
} 
Vemos que em todos os estudos o componente de mudança tecnológica é similar, girando em torno de $12 \%$ da realocação de postos de trabalho. É interessante notar que os trabalhos citados empregam fontes significativamente diferentes, sendo Hamermesh et al. (1996) apenas um biênio (1989-1990) para toda a economia holandesa e Dunne et al. (1997) e Salvanes e Forre (2003) para a média de vários anos, mas apenas para a indústria de transformação dos EUA e Noruega, respectivamente.

Deve-se destacar que a decomposição apresentada é viável apenas para bases de dados que unam trabalhadores e empresas, acompanhando-os ao longo do tempo. A RAIS permite tal acompanhamento no Brasil, ao contrário de bases de dados domiciliares (PME, PNAD, do IBGE, por exemplo) ou de pesquisas setoriais (PIA, por exemplo).

Antes de passar para a descrição da base de dados, ressalta-se que, para o caso brasileiro, o período em estudo (a década de 1990) é de grande importância, com grandes mudanças na economia. O período foi marcado por uma redução significativa no emprego industrial. A literatura debate fortemente quais as fontes da redução, a saber, choques de demanda, gerados pela abertura (câmbio valorizado), e/ou progresso tecnológico (Maia, 2003; Ribeiro et al., 2004). Ao mesmo tempo, o período foi marcado por um aumento da desigualdade salarial entre trabalhadores qualificados e não qualificados. Alguns autores atribuem isso ao progresso técnico viesado a favor da qualificação (skill biased technical change como em DeFerranti et al., 2002), embora esta visão não seja unânime (Menezes-Filho, Terra e Gonzaga, 2007). Ao lançar luz sobre as mudanças da estrutura ocupacional na economia brasileira, o trabalho pode contribuir com o debate, embora não seja objetivo do trabalho separar as fontes da redução do emprego na indústria brasileira nos anos 1990. A contribuição será indireta e poderá fomentar outros trabalhos.

\section{BASE DE DADOS}

A Base de Dados a ser usada no trabalho será a base de registros administrativos do Relatório Anual de Informações Sociais (RAIS) do Ministério do Trabalho e Emprego (MTE), para os anos de 1991 a 2000. Em conjunto com o IPEA, a base de dados está sendo tratada e empregada para estudos sobre o mercado de trabalho. O tratamento busca identificar inconsistências e possíveis variações espúrias por mudanças da cobertura ao longo do tempo. Trabalhos anteriores com a base trataram a criação e destruição de emprego (Ribeiro et al., 2004; Corseuil e Servo, 2006).

A partir das informações brutas da RAIS, é possível montar uma base de dados longitudinal, acompanhando empresas e trabalhadores ao longo do tempo. $\mathrm{O}$ in- 
dexador de trabalhadores é o PIS e o indexador das empresas, o CGC. ${ }^{10}$ A base traz dados dos trabalhadores (idade, escolaridade) e da empresa (tamanho, setor e UF). Estes dados podem ser cruzados para avaliar como as taxas de rotatividade e realocação mudam de acordo com as características das empresas e trabalhadores. Corseuil e Servo (2006) refletem um esforço de crítica e limpeza da base de dados, incorporados aqui.

Para cálculo dos fluxos de trabalhadores, foram identificados o estoque de trabalhadores no início do ano e o estoque no final do mesmo, para construir o emprego médio na empresa $x_{i t}$. Computaram-se os trabalhadores admitidos no período e os desligados, a partir do cruzamento das informações dos trabalhadores com as empresas. Com os dados das ocupações daqueles trabalhadores em $1^{\circ}$ de janeiro e 31 de dezembro, estudou-se a variação da composição ocupacional na empresa. A classificação ocupacional foi a da $\mathrm{CBO}$, embora seja possível usar o grau de escolaridade do trabalhador, para interpretações alternativas de "estrutura ocupacional”, como em Orellano e Pazzello (2006). Foi usada a CBO com classificação em três dígitos. Os dados cobrem o período de 1996 a 2002. As estimativas foram produzidas em conjunto com Carlos Henrique Corseuil. Definições diferentes para as variáveis são reportadas, com a mesma base bruta, em Corseuil e Hayashi (2006) e Corseuil (2007).

\section{RESULTADOS}

Antes de iniciar a análise, vale a pena relembrar o escopo de nossas definições. Emprego é considerado como um posto de trabalho ocupado, medido como vínculo em 31/12 na RAIS, por estabelecimento. Uma mudança de emprego em um estabelecimento representa uma mudança no número de vínculos de um ano para outro. Mudanças na estrutura ocupacional (distribuição das ocupações no emprego do estabelecimento) ou entrada e saída de trabalhadores que não alteram o estoque de empregados não são computados como mudanças de emprego. O posto de trabalho é considerado como um vínculo identificado pela ocupação referente. Variações no número de postos de trabalho de um estabelecimento são calculadas pela diferença do número de vínculos na RAIS que possuem a mesma $\mathrm{CBO}$ (três dígitos) entre um ano e outro. Troca de trabalhadores em uma mesma CBO não será computada nem como PC nem como PD. Simetricamente, uma promoção no estabelecimento, com troca de CBO para o trabalhador será calculado como uma criação e uma destruição de posto de trabalho. Por fim, a rotatividade indica a soma de admissões e desligamentos de um estabelecimento. Se na empresa há duas admissões e dois desligamentos, sem alteração de composição de CBOs no emprego do estabelecimento, e, é

\footnotetext{
${ }^{10} \mathrm{O}$ uso destes indexadores exige cuidados pois, encontra-se na base de dados múltiplos indexadores de pessoas e empresas. Estes indexadores são criados a partir de criptografia de PIS e CGC, para impedir a identificação de empresas e trabalhadores.
} 
claro, sem alteração no número de vínculos de um ano para outro, a rotatividade será igual a $4 / X$, e JC, PC, JD e PD serão iguais a zero.

Conforme a Tabela 7 , vemos que para uma variação líquida no número de empregos (postos de trabalho ocupados), $\Delta n_{t}$, de 2,6\% em média no período, $18 \%$ dos empregos do ano (média bianual) são empregos que não existiam no ano anterior (JC) e 15,4\% dos empregos foram destruídos (JD). Isto leva a uma realocação de postos de trabalho de cerca de $1 / 3$ dos empregos da economia em cada ano (GJR), como visto em Corseuil e Servo (2006).

Tabela 7: Criação, destruição e realocação de emprego, postos de trabalho e trabalhadores

\begin{tabular}{|c|c|c|c|c|c|c|c|c|}
\hline Ano & $J C$ & $J D$ & $D N$ & $G J R$ & $P C$ & $P D$ & $P R$ & $T$ \\
\hline 1996 & $17,0 \%$ & $16,5 \%$ & $0,6 \%$ & $33,5 \%$ & $23,7 \%$ & $23,1 \%$ & $46,8 \%$ & $106,4 \%$ \\
\hline 1997 & $17,5 \%$ & $16,1 \%$ & $1,5 \%$ & $33,6 \%$ & $23,8 \%$ & $22,3 \%$ & $46,1 \%$ & $109,2 \%$ \\
\hline 1998 & $17,7 \%$ & $15,8 \%$ & $1,8 \%$ & $33,5 \%$ & $23,9 \%$ & $22,0 \%$ & $45,9 \%$ & $105,2 \%$ \\
\hline 1999 & $18,3 \%$ & $15,3 \%$ & $3,0 \%$ & $33,6 \%$ & $24,4 \%$ & $21,5 \%$ & $45,9 \%$ & $101,8 \%$ \\
\hline 2000 & $19,1 \%$ & $13,6 \%$ & $5,5 \%$ & $32,8 \%$ & $25,3 \%$ & $19,8 \%$ & $45,2 \%$ & $109,5 \%$ \\
\hline 2001 & $18,4 \%$ & $14,9 \%$ & $3,5 \%$ & $33,3 \%$ & $25,1 \%$ & $21,6 \%$ & $46,6 \%$ & $113,8 \%$ \\
\hline Média & $18,0 \%$ & $15,4 \%$ & $2,6 \%$ & $33,4 \%$ & $24,4 \%$ & $21,7 \%$ & $46,1 \%$ & $107,6 \%$ \\
\hline d.p. & $0,8 \%$ & $1,0 \%$ & $1,8 \%$ & $0,3 \%$ & $0,7 \%$ & $1,1 \%$ & $0,6 \%$ & $4,2 \%$ \\
\hline
\end{tabular}

Fonte: cálculo do autor baseado em microdados da RAIS.

Considerando a criação líquida de empregos em cada empresa por categoria ocupacional (PC), vemos que aproximadamente $24 \%$ do emprego de cada ano estão em postos que foram criados naquele ano. Vale a pena reforçar as diferenças entre PC e JC. Considere uma empresa que ocupa apenas trabalhadores classificados como "diretor" e "auxiliar administrativo" e entre o ano $t$ e o ano $t$-1 o número de "diretores" aumentou em dois vínculos registrados na RAIS e o número de "auxiliar administrativo" caiu em um vínculo. A expansão do emprego líquido na empresa foi de um emprego, enquanto dois postos de trabalho foram criados.

A realocação de postos de trabalho (PR) chegou a $46 \%$ dos empregos da economia. Isto sugere que de cada dois postos de trabalho existentes em um ano, aproximadamente um foi criado ou destruído no ano. Por fim, a rotatividade de trabalhadores estimada neste trabalho chegou a mais de $100 \%$, o que pode significar que houve mais troca em emprego por parte de trabalhadores do que há empregos na economia. Se, por um lado sabemos que nem todo trabalhador realizou uma troca de emprego em um ano qualquer, a estimativa sugere que há trabalhadores que trocam de emprego várias vezes no ano.

Passando para a nossa decomposição principal (eq. 10 acima), os resultados aparecem na Tabela 8 abaixo. Vemos que, do total da rotatividade de trabalhadores da economia, a maior parte $(57 \%)$ se deve a problemas de vínculo entre empregador e empregado (T-PR), pois deu-se dentro de estabelecimentos que não alteraram a estrutura ocupacional de sua força de trabalho, nem mudaram o tamanho de sua força de trabalho. Mudanças na estrutura ocupacional podem ser interpre- 
tadas como mudanças organizacionais e/ou mudanças tecnológicas, respondem por cerca de $12 \%$ da rotatividade de trabalhadores (PR-GJR). Esta parcela de realocação dentro da empresa é menor que a realocação entre empresas $\left(G R J-\left|\Delta n_{t}\right|\right)$, que respondem por cerca de $29 \%$ da rotatividade. Esta parte da rotatividade pode ser atribuída à heterogeneidade das condições de demanda por trabalho em cada empresa. Por fim, com a menor contribuição para a rotatividade, com apenas 2,5\%, encontra-se a expansão agregada da economia $\left(\left|\Delta \mathrm{n}_{\mathrm{t}}\right|\right)$.

Tabela 8: Decomposição da rotatividade de trabalhadores no Brasil

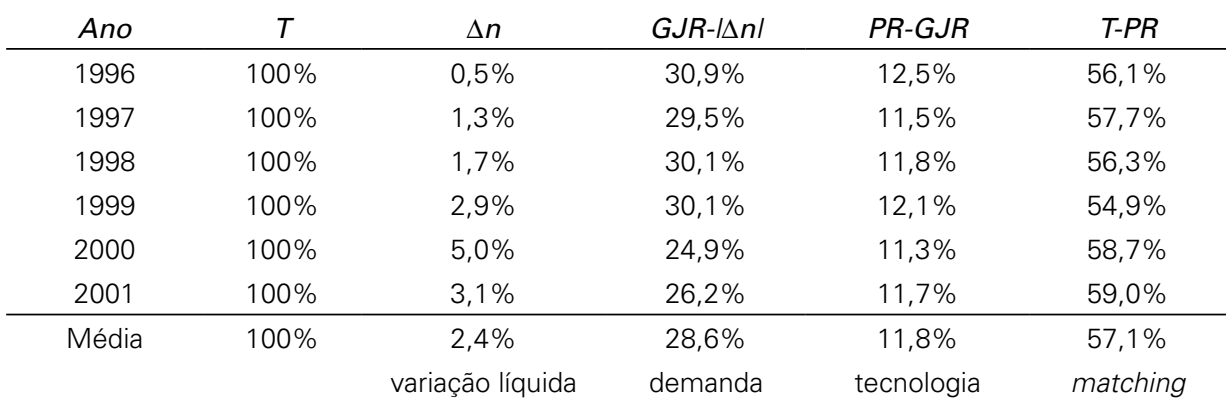

Fonte: Tabela 7 e eq. (10).

Comparando com os resultados da Tabela 5, vemos que a medida de rotatividade devido à mudanças na estrutura ocupacional no Brasil é bem maior do que em países da Europa indicados na tabela.

Os resultados da Tabela 9 trazem a decomposição (12). Concentra-se foco em aspectos de demanda por trabalho para entender a movimentação de trabalhadores (produção, choques agregados e tecnologia). As estimativas indicam que um pouco mais de $1 / 4$ da realocação de postos de trabalho por ser atribuído a mudanças ocupacionais (tecnológicas). Este valor é maior que o encontrado na literatura (vide Tabela 6 acima) e sugere que os estabelecimentos no Brasil exercem em grande parte um constante ajustamento da estrutura de sua força de trabalho por mudanças na estrutura ocupacional.

Tabela 9: Decomposição da realocação de postos de trabalho no Brasil

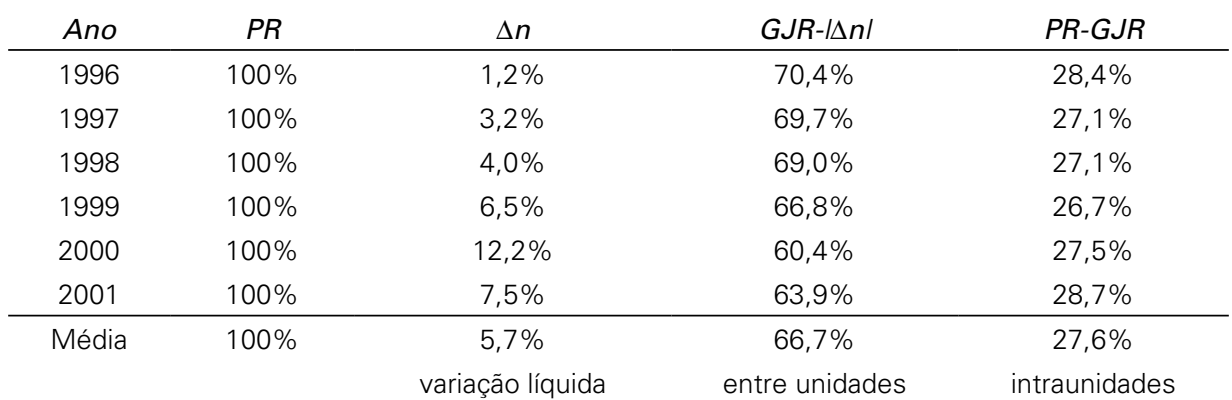

Fonte: Tabela 7 e eq. (12). 


\section{COMENTÁRIOS FINAIS}

O mercado de trabalho está em constante ebulição, principalmente no Brasil, onde a realocação de postos de trabalho e a rotatividade de trabalhadores estão entre as mais altas do mundo (Gonzaga, 2005).

Nesta pesquisa, ao considerar uma classificação de ocupações dentro de estabelecimentos (unidades econômicas), foi possível apresentar medidas de realocação de emprego, postos de trabalho e trabalhadores, de forma integrada, a partir da RAIS. Estas medidas permitiram uma interpretação dos determinantes da realocação de trabalhadores ou de postos de trabalho. Argumentou-se que uma medida de rotatividade pode ser decomposta em parcelas que dependem (i) da mudança de estrutura ocupacional dos trabalhadores da unidade, que por sua vez está associada a mudanças tecnológicas, (ii) de choques de demanda agregados, (iii) de choques de demanda idiossincráticos, específicos da unidade produtiva, e (iv) uma parcela devido a questões de vínculos (matching) — reconhecidamente residual - que pode ser devido à problemas de revelação de vínculos mais permanentes entre empresa e trabalhador.

Baseado nos dados de trabalhadores da RAIS, que envolvem mais de $17 \mathrm{mi}-$ lhões de vínculos por ano, para o período 1996-2001, concluímos que a parcela da rotatividade de trabalhadores devido a mudanças organizacionais é superior à de outros países, estando na faixa de $12 \%$ dos vínculos ativos em um ano. Estas mudanças organizacionais, ou realocação intrafirma, são responsáveis por $27 \%$ da realocação de postos de trabalho na economia.

Os resultados podem ser interpretados como indicação de que as empresas brasileiras estão engajadas, na média, em fortes processos de reestruturação e evolução, concomitantes à sua evolução em termos de tamanho. Este processo parece ser mais intenso do que em outros países e deve ser estudado em maior profundidade em futuros trabalhos.

\section{REFERÊNCIAS BIBLIOGRÁFICAS}

ABOWD, J., CORBEL, P. e KRAMARZ, F. (1999) "The entry and exit of workers and the growth of employment: and analysis of French establishments". Review of Economic and Statistics 81(2), 170-187.

ALBAEK, k. e SORENSEN, B.E. (1998) "Worker flows and job flows in Danish manufacturing, 1980-91”, The Economic Journal, 108: 1750-1771.

ANDERSON, P. e MEYER, B. (1994). The extent and consequences of job turnover. Brookings Papers: Microeconomics, 6: 177-248.

BAUER, T.K. e BENDER, S. (2004) “Technological change, organizational change, and job turnover", Labour Economics, 11 (3): 265-291.

BLANCHARD and DIAMOND (1990). "The cyclical behaviour of the gross flows of US workers". Brookings Papers: Macroeconomics, 2: 85-143.

BURGUESS, et al. (2001). "Churning dynamics". Labour Economics. v.8: 1-14.

CAMARGO, J.M. (1996). "Flexibilidade e produtividade do mercado de trabalho brasileiro" in Camargo, J.M. (org.) Flexibilidade do mercado de trabalho brasileiro, Rio de Janeiro: FGV. 
CORSEUIL, C.H. (2008) Organizational Change and Firm Dynamics. Seminários Economia PUC-Rio, $25 / 09 / 08$.

(2007) Testing the connection between replacement and job flows. Encontro nacional da ANPEC 2007, Recife.

CORSEUIL, C.H.e HAYASHI, F. (2006) "New evidences on what job creation and job destruction represent". RIETI Discussion Paper Series 06-E-023.

CORSEUIL, C.H. e SERVO, L.S. (2006) Criação, Destruição e Realocação de Empregos no Brasil. Brasília: IPEA.

DAVIS S.J. e HALTIWANGER, J. (1992) “Gross job creation, gross job destruction and employment reallocation" Quarterly Journal of Economics 106, 819-63.

(1995) "Measuring Gross Worker and Job Flows," in Labor Statistics Measurement Issues, (ed.) Haltiwanger, Manser and Topel, Chicago: University of Chicago Press.

DAVIS S.J., HALTIWANGER, J. e SCHUH, S. (1996) Job Creation and Job Destruction. Cambridge, MA: MIT Press.

DE FERRANTI, D e PERRY, G.(2002) Closing the Gap in Education and Technology. World Bank. (Ch.2-4).

DUNNE, T. et al. (1997) “Technology and jobs: secular changes and cyclical dynamics”. Carnegie-Rochester Conference Series on Public Policy v 46: 107-178.

EHRENBERG, R. e SMITH, R. (2000) A Moderna Economia do Trabalho: Teoria e Política Pública, São Paulo: Makron Books, $5^{\text {a }}$. edição.

GONZAGA, G. (2003)."Labor turnover and labor legislation in Brazil”. Economia, Journal of the LACEA 4 (1): 165-222.

GREEN, F., DICKERSON, A. e ARBACHE, J. (2001) "A picture of wage inequality and the allocation of labor through a period of trade liberalization: the case of Brazil". World Development.29 (11): 1923-1939.

HAMERMESH, D. (1993) Labor Demand. Princeton: Princeton University Press.

HAMERMESH, D., HASSINK, W.H.J. e VANOURS, J. (1996) “Job turnover and labor turnover: A taxonomy of employment dynamics”, Annales d'Économie et de Statistique, 41/42, 21-40.

HOEK, J. (2002). "Labor market institutions and restructuring: evidence from regulated and unregulated labor markets in Brazil". The William Davidson Institute Working Paper 484.

MAIA, K. (2003) "Os impactos do comércio internacional, da mudança tecnológica e da demanda final na estrutura de emprego no Brasil, 1985-1995". Revista de Economia Aplicada 7(2) 327-357.

MENEZES-FILHO, N., GONZAGA, G., and TERRA, C. (2007) "Trade liberalization and the evolution of skill earnings differentials in Brazil". Journal of International Economics, 68 (2): 345-367

MORTENSEN, D. e PISSARIDES, C. (1999) "Job reallocation, employment fluctuations and unemployment”. In J. Taylor and M.Woodford (eds) Handbook of Macroeconomics, v.I. Amsterdan: Elsevier.

MTb (1998). Manual da RAIS e CAGED. Informações CGETIP. Brasília:MTb.

MTb/IPEA (1995) Mercado de Trabalho: conjuntura e análise - Balanço de 1995. Brasília: IPEA.

NERI, M. et al. (1996) “Aspectos dinâmicos do desemprego e da ocupação”. Estudos Econômicos.

ORELLANO, V. e PAZELLO, E. (2006) "Evolução e determinantes da rotatividade da mão-de-obra nas firmas da indústria paulista na década de 1990”. Pesquisa e Planejamento Econômico, 36 (1): 179-207.

PAZELLO, E., BIVAR, W. e GONZAGA, G. (2000) "Criação e destruição de postos de trabalho por tamanho da empresa no Brasil”. Pesquisa e Planejamento Econômico, 30 (2): 259-288.

RIBEIRO, E. P. et al. (2004) Trade liberalization, the exchange rate, and job flows in Brazil. Journal of Policy Reform, Londres, 7 (4): 219-233.

SALVANES, K. E FORRE, S. (2003) "Effects on employment of trade and technical change: Evidence from Norway", Economica 70: 293-329.

WORLDBANK (2002). Brazil Jobs Report. Washington, DC: WorldBank. 